\title{
A solution of the minimum-time velocity planning problem based on lattice theory
}

\author{
Luca Consolini $^{1}$, Mattia Laurini ${ }^{1}$, Marco Locatelli ${ }^{1}$, Andrea Minari ${ }^{1}$ \\ ${ }^{1}$ Dipartimento di Ingegneria e Architettura, Università degli Studi di Parma, \\ Parco Area delle Scienze 181/A, 43124 Parma, Italy. \\ luca.consolini@unipr.it, mattia.laurini@unipr.it,marco.locatelli@unipr.it, andrea.minari2@studenti.unipr.it
}

\begin{abstract}
For a vehicle on an assigned path, we find the minimum-time speed law that satisfies kinematic and dynamic constraints, related to maximum speed and maximum tangential and transversal acceleration. We present a necessary and sufficient condition for the feasibility of the problem and a simple operator, based on the solution of two ordinary differential equations, which computes the optimal solution. Theoretically, we show that the problem feasible set, if not empty, is a lattice, whose supremum element corresponds to the optimal solution.
\end{abstract}

Index terms - Optimal Control, Speed Planning, Minimum-Time Problems, Lattice Theory, Autonomous Vehicles

\section{Introduction}

An important problem in motion planning is the computation of the minimum-time motion of a carlike vehicle from a start configuration to a target one while avoiding collisions (obstacle avoidance) and satisfying kinematic, dynamic and mechanical constraints (for instance, on velocities, accelerations and maximal steering angle). This problem can be approached in two ways:

1. as a minimum-time trajectory planning where both the path to be followed by the vehicle and the timing law on this path (i.e., the vehicle's velocity) are simultaneously designed; or

2. as a (geometric) path planning followed by a minimum-time velocity planning on the planned path (see for instance [8]).

In this paper, following the second paradigm, we assume that a path that joins the initial and final configurations, compatible with the maximum curvature allowed for the car-like vehicle, is assigned and we aim at finding the time-optimal speed law that satisfies some kinematic and dynamic constraints.

Previous works address the problem in time domain. For instance, [16] investigates necessary optimality conditions that allows deriving a semi-analytical solution. Reference [7] assumes that the vehicle moves along a specified clothoid and proposes a semi-analytical solution for the optimal profile of the longitudinal acceleration. Some approaches propose special speed profiles that guarantee the satisfaction of kinematic and dynamic constraints (see for instance [11, 15, 18, 3]). Other works 
(see, for instance, [2, 17, 14, 10, 12]) represent the speed law as a function $v$ of the arc-length position $s$ and not as a function of time.

Here, we follow this second approach, that considerably simplifies the optimization problem. This paper is a development of our previous works $([4,5])$, that consider the following problem

$$
\begin{array}{rlr}
\min _{v \in W^{1, \infty}\left(\left[0, s_{f}\right]\right)} \int_{0}^{s_{f}} v^{-1}(s) d s & \\
& v^{-}(s) \leq v(s) \leq v^{+}(s), & \\
& \alpha^{-}(s) \leq 2 v^{\prime}(s) v(s) \leq \alpha^{+}(s), & s \in\left[0, s_{f}\right], \\
& |k(s)| v(s)^{2} \leq \beta(s), & s \in\left[0, s_{f}\right],
\end{array}
$$

Here, $v^{-}, v^{+}, \alpha^{-}, \alpha^{+}$are assigned functions, with $v^{-}, v^{+}$non-negative. The objective function (1a) is the total maneuver time and constraints (1b), (1c), (1d) limit velocity and the tangential and normal components of acceleration.

Problem (1) belongs to a class of problems that includes optimal velocity planning for manipulators and has the form

$$
\begin{aligned}
\min _{v \in W^{1, \infty}\left(\left[0, s_{f}\right]\right)} & \int_{0}^{s_{f}} v^{-1}(s) d s \\
& a_{i}(s) \dot{v}(s) v(s)+b_{i}(s) v(s)^{2}+c_{i}(s) \leq 0, i=1, \ldots, m,
\end{aligned}
$$

where $m$ is the number of constraints and $a_{i}, b_{i}, c_{i}$ are assigned functions. It is clear that Problem (1) is a special case of Problem (2), obtained for a specific choice of $a_{i}, b_{i}, c_{i}$.

Our previous works [4, 5] present an algorithm, with linear-time computational complexity with respect to the number of variables, that provides an optimal solution of (1) after spatial discretization. Namely, the path is divided into $n$ intervals of equal length and Problem (1) is approximated with a finite dimensional one in which the derivative of $v$ is substituted with a finite difference approximation.

In this paper, we compute directly the exact continuous-time solution of Problem (1) without performing a finite-dimensional reduction. The main result of the paper is presented in Theorem 3.1 . It gives a sufficient and necessary condition for the feasibility of Problem (1) and presents its optimal solution, which is computed as the pointwise minimum of the solutions of two ODEs.

The method we propose presents some resemblances with the method of "numerical integration", introduced for problems of class (2). For instance, [14] proposes a method, based on the identification of "characteristic switching points" in which the maximum velocity is attained. This simplifies the calculation of the optimal velocity profile. A related algorithm is presented in [9]. Recent paper [13] presents various properties of numerical integration methods. Anyway, the method we propose is simpler and more efficient since it leverages the special structure of Problem (1) with respect to the more general problem (2).

Statement of contribution: With respect to existing literature, the new contributions of this work are the following ones:

- It presents a necessary and sufficient condition for the feasibility of Problem (1) (see part $i$. of Theorem 3.1.

- It proposes a simple operator, based on the solution of two ordinary differential equations, that computes the optimal solution (see part ii. of Theorem 3.1). 
Note that these results correspond to the generalization to the continuous-time case of the results presented in [5] for the spatially-discretized version of Problem (1). In fact, this paper shares some of its fundamental ideas with [5]. Namely:

- The feasible set of Problem (1) has the algebraic structure of a lattice, if equipped with the operations of pointwise minimum and maximum.

- The optimal solution of Problem (1) corresponds to the supremal element of this lattice.

- The optimal solution of Problem (1) is obtained with a projection operation and its optimality is proven by the Knaster-Tarski Fixpoint Theorem.

Anyway, solving the problem in a function space requires various nontrivial technical extensions to the proofs presented in [5].

Notation: Given an interval $I=[a, b]$ and a measurable function $f: I \rightarrow \mathbb{R}$, let us recall that

$$
\begin{gathered}
\|f\|_{\infty}=\inf \{C \geq 0:|f(x)| \leq C \text { for almost every } x \in I\}, \\
L^{\infty}(I)=\left\{f: I \rightarrow \mathbb{R}:\|f\|_{\infty}<\infty\right\},
\end{gathered}
$$

and

$$
W^{1, \infty}(I)=\left\{f \in L^{\infty}(I): D f \in L^{\infty}(I)\right\},
$$

where $D f$ denotes the weak derivative of $f$. Let $f, g: I \rightarrow \mathbb{R}$, define $f \wedge g, f \vee g$, as, respectively, the pointwise minimum and maximum operations; moreover let us define the partial order $\leq$ as follows

$$
f \leq g \Longleftrightarrow \forall x \in I f(x) \leq g(x) .
$$

We will write "almost everywhere" as "a. e." and we will use symbol $\not$ at the end of a proof to state that a contradiction has been reached.

Paper organization: Section 2 presents the addressed optimal control problem. Section 3 presents the main result (Theorem 3.1) and Section 4 presents some examples. Finally, Section 5 presents the proof of the main result.

\section{Problem formulation}

Let $\gamma:\left[0, s_{f}\right] \rightarrow \mathbb{R}^{2}$ be a $C^{2}$ function such that $\left\|\gamma^{\prime}(\lambda)\right\|=1, \forall \lambda \in\left[0, s_{f}\right]$. The image set $\gamma\left(\left[0, s_{f}\right]\right)$ represents the path followed by a vehicle, $\gamma(0)$ the initial configuration and $\gamma\left(s_{f}\right)$ the final one. We want to compute the speed-law that minimizes the overall transfer time while satisfying some kinematic and dynamic requirements. To this end, let $\lambda:\left[0, t_{f}\right] \rightarrow\left[0, s_{f}\right]$ be a differentiable monotone increasing function that represents the vehicle position as a function of time and let $v:\left[0, s_{f}\right] \rightarrow[0,+\infty]$ be such that, $\forall t \in\left[0, t_{f}\right], \dot{\lambda}(t)=v(\lambda(t))$. In this way, $v(s)$ is the vehicle velocity at position $\mathrm{s}$. The position of the vehicle as a function of time is given by $x:\left[0, t_{f}\right] \rightarrow \mathbb{R}^{2}, x(t)=\gamma(\lambda(t))$, the velocity and acceleration are given by

$$
\begin{gathered}
\dot{x}(t)=\gamma^{\prime}(\lambda(t)) v(\lambda(t)), \\
\ddot{x}(t)=a_{L}(t) \gamma^{\prime}(\lambda(t))+a_{N}(t) \gamma^{\perp}(\lambda(t)),
\end{gathered}
$$

where $a_{L}(t)=v^{\prime}(\lambda(t)) v(\lambda(t))$ and $a_{N}(t)(t)=k(\lambda(t)) v(\lambda(t))^{2}$ are, respectively, the longitudinal and normal components of acceleration. Here $k:\left[0, s_{f}\right] \rightarrow \mathbb{R}$ is the scalar curvature, defined as $k(s)=$ $\left\langle\gamma^{\prime \prime}(s), \gamma^{\prime}(s)^{\perp}\right\rangle$. 


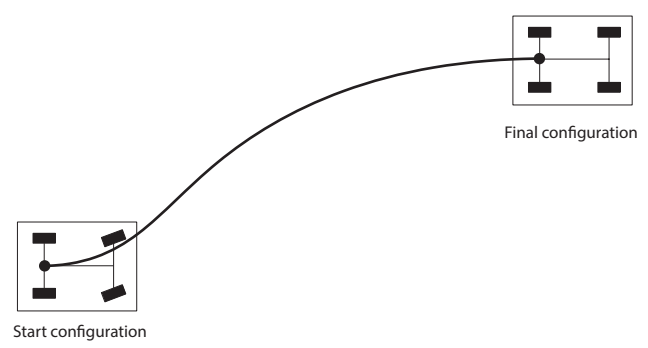

Figure 1: A path to follow for an autonomous car-like vehicle.

We require to travel the distance $s_{f}$ in minimum-time while satisfying constraints on the vehicle velocity and on its longitudinal and normal acceleration. The minimum-time problem can be approached by searching a velocity profile $v$ which is the solution of Problem (1).

It is convenient to make the change of variables $w=v^{2}$ (see also [17]), so that Problem (1) takes on the form

$$
\begin{array}{rlr}
\min _{w \in W^{1, \infty}\left(\left[0, s_{f}\right]\right)} & \int_{0}^{s_{f}} w(s)^{-\frac{1}{2}} d s & \\
& \mu^{-}(s)<w(s) \leq \mu^{+}(s), & \\
& \alpha^{-}(s) \leq w^{\prime}(s) \leq \alpha^{+}(s), & s \in\left[0, s_{f}\right], \\
& s \in\left[0, s_{f}\right],
\end{array}
$$

where

$$
\mu^{+}(s)=v^{+}(s)^{2} \wedge \frac{\beta(s)}{k(s)}, \quad \mu^{-}(s)=v^{-}(s)^{2}
$$

represent the upper bound of $w$, (depending on the velocity bound $v^{+}$and the curvature $k$ ) and the lower bound of $w$, respectively.

In this paper, we actually address the following problem, which is slightly more general than (3),

$$
\begin{array}{rlrl}
\min _{w \in W^{1, \infty}\left(\left[0, s_{f}\right]\right)} \Psi(w) & & \\
& \mu^{-}(s)<w(s) \leq \mu^{+}(s), & & s \in\left[0, s_{f}\right], \\
& \alpha^{-}(s) \leq w^{\prime}(s) \leq \alpha^{+}(s), & & s \in\left[0, s_{f}\right],
\end{array}
$$

where $\Psi: W^{1, \infty}\left(\left[0, s_{f}\right]\right) \rightarrow \mathbb{R}$ is order reversing (i.e., $\left.x \geq y \Rightarrow \Psi(x) \leq \Psi(y)\right)$ and $\mu^{-}, \mu^{+}, \alpha^{-}, \alpha^{+} \in$ $L^{\infty}\left(\left[0, s_{f}\right]\right)$ are assigned functions with $\mu^{-}, \alpha^{+} \geq 0, \alpha^{-} \leq 0$. Note that the objective function (3a $)$ is order reversing, so that Problem (3) has the form (5). Consider the following:

Definition 2.1. Let $\mathscr{Q}$ be the subset of $W^{1, \infty}\left(\left[0, s_{f},\right]\right)$ such that $\mu \in \mathscr{Q}$ if $\operatorname{sign}\left(\mu^{\prime}-\alpha^{+}\right)$and $\operatorname{sign}\left(\mu^{\prime}-\alpha^{-}\right)$ are Riemann integrable (i.e., in view of the boundedness of the sign function, a. e. continuous), where sign $: \mathbb{R} \rightarrow\{-1,0,1\}$ is defined as

$$
\operatorname{sign}(x)= \begin{cases}1, & \text { if } x>0 \\ 0, & \text { if } x=0 \\ -1, & \text { if } x<0\end{cases}
$$




\section{Main Results}

Define the forward operator $F: \mathscr{Q} \rightarrow W^{1, \infty}\left(\left[0, s_{f}\right]\right)$ such that $F(\mu)=\phi$, where $\phi$ is the solution of the following differential equation

$$
\begin{cases}\phi^{\prime}(x)=f(x, \phi)= \begin{cases}\alpha^{+}(x) \wedge \mu^{\prime}(x), & \text { if } \phi(x) \geq \mu(x) \\ \alpha^{+}(x), & \text { if } \phi(x)<\mu(x)\end{cases} \\ \phi(0)=\mu(0) .\end{cases}
$$

Note that the solution of (6) exists and is unique by Theorem 1 in Chapter 2, Section 10 of [1], since function $f$ is bounded on $\left[0, s_{f}\right]$, the subset of $\left[0, s_{f}\right] \times \mathbb{R}$ in which $f$ is discontinuous has zero measure and, $\forall x \in\left[0, s_{f}\right], \forall u, y \in \mathbb{R}$,

$$
(u-y)(f(x, u)-f(x, y)) \leq 0 .
$$

Conversely, define the backward operator $B: \mathscr{Q} \rightarrow W^{1, \infty}\left(\left[0, s_{f}\right]\right)$, such that $B(\mu)=\phi$, where $\phi$ is the solution of

$$
\begin{cases}\phi^{\prime}(x) & = \begin{cases}\alpha^{-}(x) \vee \mu^{\prime}(x), & \text { if } \phi(x) \geq \mu(x) \\ \alpha^{-}(x), & \text { if } \phi(x)<\mu(x)\end{cases} \\ \phi\left(s_{f}\right) & =\mu\left(s_{f}\right),\end{cases}
$$

whose existence and uniqueness hold for the same reasons as (6).

Finally, define the meet operator $M: \mathscr{Q} \rightarrow W^{1, \infty}\left(\left[0, s_{f}\right]\right)$ as

$$
M(\mu)=F(\mu) \wedge B(\mu) .
$$

We claim that the meet operator $M$ allows checking the feasibility of Problem (5) and that, in case Problem (5) is feasible, function $v^{*}=M\left(\mu^{+}\right)$represents its optimal solution.

Namely, the following is the main result of this paper.

Theorem 3.1. Let $\mu^{+} \in \mathscr{Q}$, then the following statements hold:

i. Problem (5) is feasible if and only if function $w^{*}=M\left(\mu^{+}\right)$satisfies

$$
w^{*} \geq \mu^{-} .
$$

ii. If Problem (5) is feasible, then function $w^{*}=M\left(\mu^{+}\right)$is its optimal solution.

Proofs of the results. Part $i$. follows from Proposition 5.11, part ii. follows from Proposition 5.12 (see Section 5).

\section{Examples}

As a first example consider the path shown in Figure 2, whose curvature is defined as

$$
k(s)= \begin{cases}0, & \text { if } s \in\left[0, l_{1}\right) \\ k_{\tau}(s), & \text { if } s \in\left[l_{1}, l_{2}\right] \\ 1 / R, & \text { if } s \in\left(l_{2}, l_{3}\right) \\ k_{\tau}(s), & \text { if } s \in\left[l_{3}, l_{4}\right] \\ 0, & \text { if } s \in\left(l_{4}, s_{f}\right]\end{cases}
$$



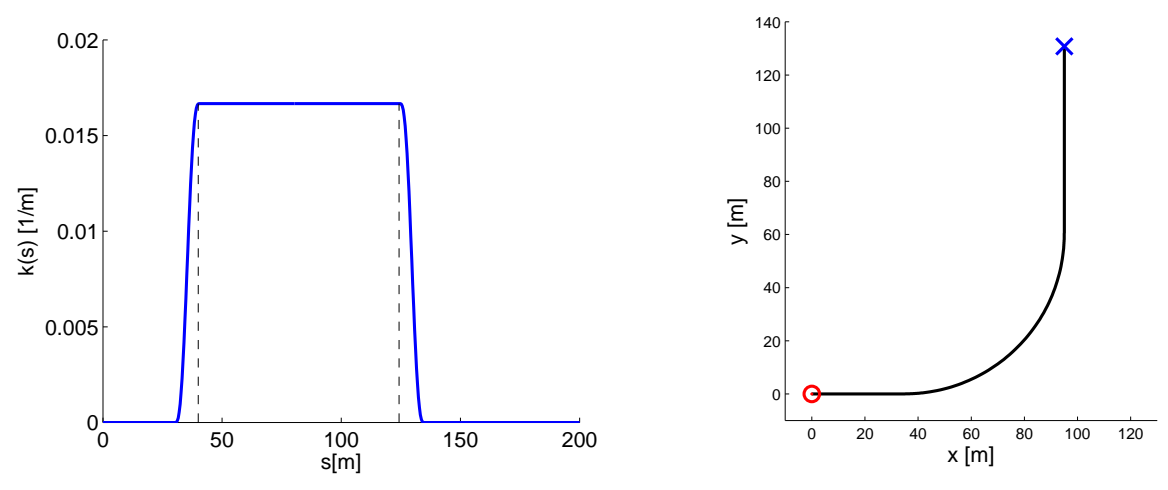

Figure 2: On the left, the curvature function $k$ in (9) of the curve discussed in the first example. On the right, the black line represents the path, the red circle and the black cross represent the starting point and, respectively, the end point.

where $k_{\tau}(s)$ is the 6-th degree Hermite polynomial used to guarantee the following interpolation conditions:

$$
\begin{aligned}
& k\left(l_{1}\right)=k\left(l_{4}\right)=0, \\
& k\left(l_{2}\right)=k\left(l_{3}\right)=1 / R, \\
& k^{\prime}\left(l_{i}\right)=k^{\prime \prime}\left(l_{i}\right)=0, \quad i=1, \ldots, 4 .
\end{aligned}
$$

In this example, the total length is $s_{f}=200$ and the minimum-time velocity planning problem is addressed with $\left[l_{1}, l_{2}, l_{3}, l_{4}\right]=[30,40,124.2478,134.2478], R=60$. The velocity bounds $v^{+}$and $v^{-}$ are set as follows: $v^{+}(0)=v^{-}(0)=0, v^{+}\left(s_{f}\right)=v^{-}\left(s_{f}\right)=22$, while, for each $s \in\left(0, s_{f}\right), v^{-}(s)=0$ and $v^{+}(s)=36.1$. The longitudinal acceleration limits are $\alpha^{-}=-10.5$ and $\alpha^{+}=4$, and the maximal normal acceleration is $\beta=7$.

The following results are obtained by numerically solving equations (6), (7) with a standart RungeKutta 45 integration scheme. Figure 3 shows the upper-bound function $\mu^{+}$obtained by (4) and the corresponding functions $F(u)$ and $B(u)$ computed as the solution of equations (6) and (7), respectively. Figure 4 shows the optimal solution $w^{*}$ obtained with (8). In this example, the vehicle starts with zero velocity and accelerates to the upper bound. Then, it follows the velocity bound in order to respect the maximum velocity constraint due to the lateral acceleration on the curve. After that, at the end of the constant bound, the vehicle accelerates and reaches a second local maximum velocity after which it decelerates quickly in order to reach the final velocity $v^{+}\left(s_{f}\right)$.

As a second example, consider the same path and constraints as in the first example, with different initial and final conditions: $v^{-}(0)=v^{+}(0)=0, v^{-}\left(s_{f}\right)=v^{+}\left(s_{f}\right)=35$. Figure 5 shows function $w^{*}$ obtained by (8). In this case, Problem (5) is unfeasible by Theorem 3.1, being $w^{*}\left(s_{f}\right)<v^{-}\left(s_{f}\right)^{2}$. In fact, the allowed maximum longitudinal acceleration is not sufficient to reach the final condition on velocity.

As a third example, consider a curve obtained by a quintic polynomial curve which interpolates coordinates $x=[0,2,2.60,1.75,3], y=[0,-0.5,0,2,3]$ (see Figure 6). The velocity planning is addressed with $v^{+}(0)=v^{-}(0)=0, v^{+}\left(s_{f}\right)=v^{-}\left(s_{f}\right)=0$ and with $v^{-}(s)=0$ and $v^{+}(s)=1.3$ for each $s \in\left(0, s_{f}\right)$. The longitudinal acceleration limits are $\alpha^{-}=-0.1, \alpha^{+}=0.1$, and the maximal normal acceleration $\beta=0.05$. The resulting optimal velocity profile is plotted in Figure 7 


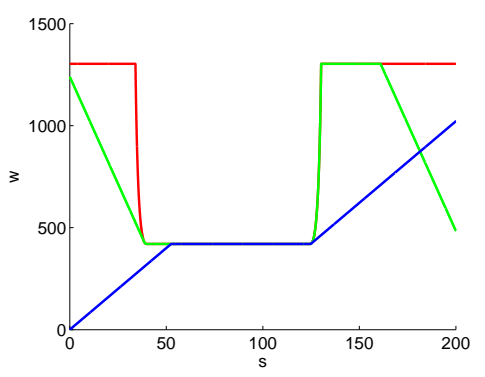

Figure 3: Example 1: The red line represents function $\mu^{+}$defined in (4), the blue line represents $F(u)$ while the green line represents $B(u)$.

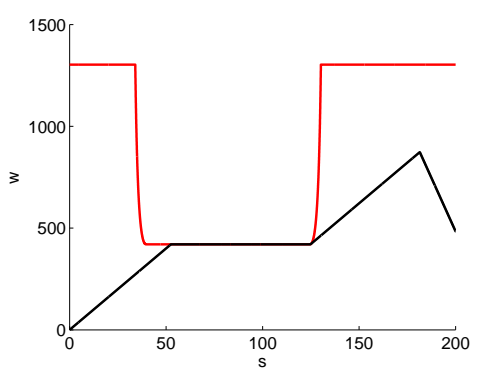

Figure 4: Example 1: The red line represents $\mu^{+}$defined in (4), the black line represents the optimal solution $w^{*}=M(u)$.

\section{Proofs}

Given the interval $I=\left[0, s_{f}\right]$, let $P=\left\{u \in L^{\infty}(I): 0 \leq u(s) \leq\left\|\mu^{+}\right\|_{\infty}\right.$ for almost every $\left.s \in I\right\}$. Note that $\langle P ; \vee, \wedge\rangle$ is a complete lattice. Hence, for each subset $S \subseteq P$, there exists a unique least upper bound $u \in P$, such that, $\forall v \in P$

$$
(\forall w \in S w \leq v) \Longleftrightarrow u \leq v .
$$

The least upper bound of $S$ is denoted by $\bigvee S$. Dually, it is possible to define the greatest lower bound of $S \subseteq P$, denoted by $\wedge S$ (see Definitions 2.1, 2.4 and Notation 2.3 on pages 33-34 of [6]).

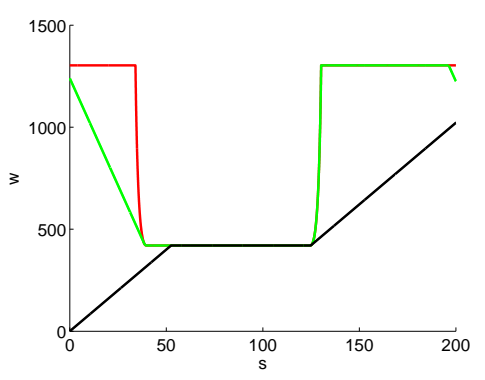

Figure 5: Example 2. The green line represents the velocity function $B(u)(s)$ while the black one depicts function $w^{*}=M(u)$. The final velocity condition is not satisfied: $w^{*}\left(s_{f}\right) \neq v_{f}^{2}$. 


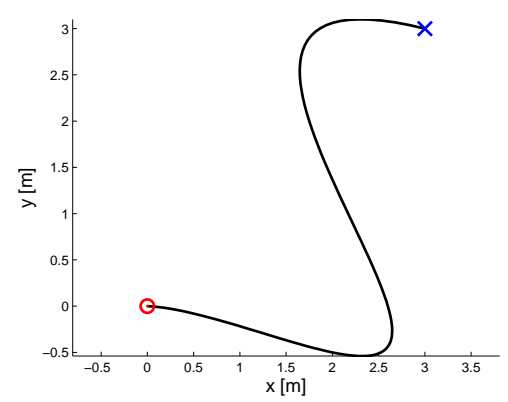

Figure 6: A path obtained by quintic-splines interpolation. The black line represents the path while the circle and the cross represent the start and the end point, respectively.

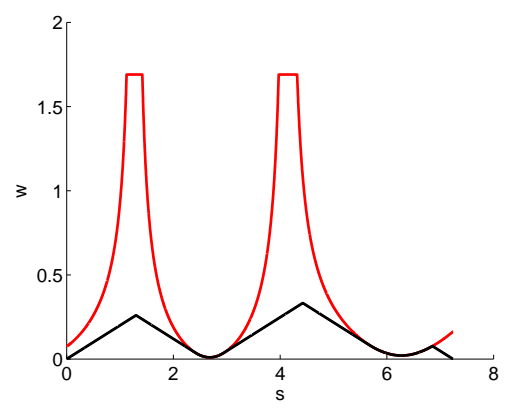

Figure 7: The red line represents function $\mu^{+}$defined in (4), the black line is the optimal velocity profile $w^{*}=M(u)$.

Given function $\chi: \mathbb{R} \rightarrow\{0,1\}$ defined as follows

$$
\chi(s)= \begin{cases}1, & \text { if } s \geq 0 \\ 0, & \text { otherwise }\end{cases}
$$

let us define $\forall x, y \in I$, function $A: I \times I \rightarrow \mathbb{R}$ as

$$
A(x, y)=\int_{x}^{y}\left\{\alpha^{+}(\xi) \chi(y-x)+\alpha^{-}(\xi) \chi(x-y)\right\} d \xi .
$$

Define, also, operators $\bar{F}, \bar{B}, \bar{M}: P \rightarrow P$, such that, for $\mu \in P, \bar{F}(\mu)$ and $\bar{B}(\mu)$ are given as follows

$$
\begin{aligned}
& \left\{\begin{array}{l}
\bar{F}(\mu)(x)=\bigwedge_{y \leq x}\{\mu(y)+A(y, x)\} \\
\bar{F}(\mu)(0)=\mu(0),
\end{array}\right. \\
& \left\{\begin{array}{l}
\bar{B}(\mu)(x)=\bigwedge_{y \geq x}\{\mu(y)+A(y, x)\} \\
\bar{B}(\mu)\left(s_{f}\right)=\mu\left(s_{f}\right) .
\end{array}\right.
\end{aligned}
$$

and $\bar{M}(\mu)=\bar{F}(\mu) \wedge \bar{B}(\mu)$. Observe that $\forall x \in I$,

$$
\bar{M}(\mu)(x)=\bigwedge_{y \leq x}\{\mu(y)+A(y, x)\} \wedge \bigwedge_{y \geq x}\{\mu(y)+A(y, x)\}=\bigwedge_{y \in I}\{\mu(y)+A(y, x)\} .
$$


As we will show in Proposition 5.9, the operators we just introduced are extensions of those defined, respectively, in (6), (7) and (8).

We will refer to the following definitions.

Definition 5.1. An operator $\phi: P \rightarrow P$ is meet preserving if, $\forall u, v \in P$,

$$
\phi(u \wedge v)=\phi(u) \wedge \phi(v) .
$$

Definition 5.2. An operator $\phi: P \rightarrow P$ is order preserving if, $\forall u, v \in P$,

$$
u \leq v \Rightarrow \phi(u) \leq \phi(v) .
$$

Even though we will only use the fact that operators $\bar{F}, \bar{B}$ and $\bar{M}$ are order preserving, for completeness we state the following Proposition.

Proposition 5.3. Operators $\bar{F}, \bar{B}$ and $\bar{M}$ are meet preserving and order preserving.

Proof. Let $u, v \in P, w=u \wedge v$. We want to show that $\bar{F}(w)=\bar{F}(u) \wedge \bar{F}(v)$. By definition of $\bar{F}$ we have that $\bar{F}(w)(0)=\bar{F}(u)(0) \wedge \bar{F}(v)(0)$ and $\forall x \in I$,

$$
\begin{aligned}
\bar{F}(w)(x) & =\bigwedge_{y \leq x}\{w(y)+A(y, x)\}=\bigwedge_{y \leq x}\{(u \wedge v)(y)+A(y, x)\}=\bigwedge_{y \leq x}\{u(y) \wedge v(y)+A(y, x)\}= \\
& =\bigwedge_{y \leq x}\{u(y)+A(y, x)\} \wedge \bigwedge_{y \leq x}\{v(y)+A(y, x)\}=\bar{F}(u)(x) \wedge \bar{F}(v)(x) .
\end{aligned}
$$

The proof that $\bar{B}(u \wedge v)=\bar{B}(u) \wedge \bar{B}(v)$ is analogous. Finally, $\bar{M}(u \wedge v)=\bar{F}(u \wedge v) \wedge \bar{B}(u \wedge v)=$ $\bar{F}(u) \wedge \bar{F}(v) \wedge \bar{B}(u) \wedge \bar{B}(v)=\bar{F}(u) \wedge \bar{B}(u) \wedge \bar{F}(v) \wedge \bar{B}(v)=\bar{M}(u) \wedge \bar{M}(v)$. Since maps $\bar{F}, \bar{B}, \bar{M}$ are meet preserving, they are also order preserving (see Proposition 2.19 on page 44 of [6]).

Proposition 5.4. Function $A: I \times I \rightarrow \mathbb{R}$ defined as in (10) is a hemi-metric, that is, it satisfies the following properties:

i. $\forall x, y \in I, A(x, y) \geq 0$,

ii. $\forall x \in I, A(x, x)=0$,

iii. $\forall x, y, z \in I, A(x, z) \leq A(x, y)+A(y, z)$ (i.e., the triangular inequality holds).

Moreover, equality holds if $x \geq y \geq z$ or $x \leq y \leq z$.

Proof. $\quad i$. It holds, since $\alpha^{+}$is non-negative and $\alpha^{-}$is non-positive over $I$.

ii. It holds trivially by definition of $A$.

iii. For $y \geq z \geq x$ :

$$
\begin{aligned}
A(x, z) & =\int_{x}^{z}\left\{\alpha^{+}(\xi) \chi(z-x)+\alpha^{-}(\xi) \chi(x-z)\right\} d \xi=\int_{x}^{z} \alpha^{+}(\xi) d \xi \leq \\
& \leq \int_{x}^{y} \alpha^{+}(\xi) d \xi \leq \int_{x}^{y} \alpha^{+}(\xi) d \xi-\int_{z}^{y} \alpha^{-}(\eta) d \eta=\int_{x}^{z} \alpha^{+}(\xi) d \xi+\int_{y}^{z} \alpha^{-}(\eta) d \eta= \\
& =\int_{x}^{y}\left\{\alpha^{+}(\xi) \chi(y-x)+\alpha^{-}(\xi) \chi(x-y)\right\} d \xi+\int_{y}^{z}\left\{\alpha^{+}(\eta) \chi(z-y)+\alpha^{-}(\eta) \chi(y-z)\right\} d \eta= \\
& =A(x, y)+A(y, z) .
\end{aligned}
$$


The same reasoning applies also to the case when $z \geq x \geq y, x \geq z \geq y$ or $y \geq x \geq z$. Next, let us show that equality holds for any $x \leq y \leq z$ :

$$
\begin{aligned}
A(x, z) & =\int_{x}^{z}\left\{\alpha^{+}(\xi) \chi(z-x)+\alpha^{-}(\xi) \chi(x-z)\right\} d \xi=\int_{x}^{z} \alpha^{+}(\xi) d \xi=\int_{x}^{y} \alpha^{+}(\xi) d \xi+\int_{y}^{z} \alpha^{+}(\eta) d \eta= \\
& =\int_{x}^{y}\left\{\alpha^{+}(\xi) \chi(y-x)+\alpha^{-}(\xi) \chi(x-y)\right\} d \xi+\int_{y}^{z}\left\{\alpha^{+}(\eta) \chi(z-y)+\alpha^{-}(\eta) \chi(y-z)\right\} d \eta= \\
& =A(x, y)+A(y, z) .
\end{aligned}
$$

The proof that the equality holds also for any $x \geq y \geq z$ is analogous.

Proposition 5.5. Function $\bar{M}$ satisfies the following properties, $\forall \mu \in P$,

i. $\bar{M}(\mu) \leq \mu$,

ii. $\bar{M}^{2}(\mu)=\bar{M}(\mu)$, where $\bar{M}^{2}(\mu)$ stands for $\bar{M}(\bar{M}(\mu))$.

Proof. $\quad i$. It is a consequence of the definition of $\bar{M}$.

ii. Let us now show that $\bar{F}(\bar{M}(\mu))=\bar{M}(\mu)$ : the fact that $\bar{F}(\bar{M}(\mu)) \leq \bar{M}(\mu)$ follows by the definition of $\bar{F}$ whilst, to prove the opposite inequality, note that, by Proposition 5.4 and (11),

$$
\begin{aligned}
\bar{F}(\bar{M}(\mu))(x) & =\bigwedge_{y \leq x}\{\bar{M}(\mu)(y)+A(y, x)\}=\bigwedge_{y \leq x}\left\{\bigwedge_{z \in I}\{\mu(z)+A(z, y)\}+A(y, x)\right\} \geq \\
& \geq \bigwedge_{z \in I}\{\mu(z)+A(z, x)\}=\bar{M}(\mu)(x) .
\end{aligned}
$$

In the same way it can be proved that $\bar{B}(\bar{M}(\mu))=\bar{M}(\mu)$, from which it follows that $\bar{M}(\bar{M}(\mu))=$ $\bar{M}(\mu)$.

\section{Proposition 5.6.}

$$
\bar{M}\left(\mu^{+}\right)=\bigvee\left\{u \in P: u \leq \bar{M}(u), u \leq \mu^{+}\right\}
$$

Proof. Set $U=\left\{u \in P: u \leq \mu^{+}\right\}$. Note that $\langle U ; \vee, \wedge\rangle$ is a sublattice of $\langle P ; \vee, \wedge\rangle$, moreover, by $i$. of Proposition 5.5, if $u \in U$, then $\bar{M}(u) \in U$. Since $\bar{M}$ is order preserving by Proposition 5.3, by the Knaster-Tarski Fixpoint Theorem (Theorem 2.35 on page 50 in [6])

$$
u^{*}=\bigvee\left\{u \in P: u \leq \bar{M}(u), u \leq \mu^{+}\right\}
$$

is such that $u^{*}$ is the greatest fixed point of $\bar{M}$ such that $u^{*} \in U$. Let $u=\bar{M}\left(\mu^{+}\right)$, by part $i i$. of Proposition 5.5, we know that $u$ is also a fixed point of $\bar{M}$, thus, by definition of $u^{*}, u^{*} \geq u$. To prove that $u^{*}=u$, that is, to prove that $u$ is also the greatest fixed point, it remains to show that $u^{*} \leq u$. To this end, assume, by contradiction, that $u^{*} \not \leq u$. Since $u^{*}=\bar{M}\left(u^{*}\right), u=\bar{M}\left(\mu^{+}\right)$and the fact that $\bar{M}$ is order preserving, it follows that $u^{*} \not \leq \mu^{+}$, which contradicts the definition of $u^{*} \downarrow$.

Remark 5.7. Given $u, v \in P$, if $u \not \leq v$, this does not imply that $u \geq v$ and $u \neq v$, as $u$ and $v$ may not be comparable with respect to partial order $\leq$. 
Proposition 5.8. The following two statements are equivalent:

i. Set $\left\{u \in P: u=\bar{M}(u), \mu^{-} \leq u \leq \mu^{+}\right\}$is not empty.

ii. $\bar{M}\left(\mu^{+}\right) \geq \mu^{-}$.

Proof.

ii. $\Rightarrow i$.) It follows from the fact that $u^{*}=\bar{M}\left(\mu^{+}\right)$is such that $\bar{M}\left(u^{*}\right)=u^{*}$ by part ii. of Proposition 5.5.

i. $\Rightarrow$ ii.) By contradiction, assume that $\bar{M}\left(\mu^{+}\right) \nsupseteq \mu^{-}$. Choose any $w \in P$ such that $w=\bar{M}(w)$ and $w \leq \mu^{+}$. By Proposition 5.6, $\bar{M}(w) \leq \bar{M}\left(\mu^{+}\right)$, hence $w \leq \bar{M}\left(\mu^{+}\right)$, but $\bar{M}\left(\mu^{+}\right) \nsupseteq \mu^{-}$, so $w \nsupseteq \mu^{-}$. Thus, being $w$ any fixed point of $\bar{M}$ such that $w \leq \mu^{+}$, set $\left\{u \in P: u=\bar{M}(u), \mu^{-} \leq u \leq \mu^{+}\right\}$is empty $\downarrow$.

Proposition 5.9. If $\mu \in \mathscr{Q}$, then $\bar{F}(\mu), \bar{B}(\mu) \in W^{1, \infty}(I)$ satisfy a. $e$.

$$
\begin{cases}\bar{F}(\mu)^{\prime}(x) & = \begin{cases}\alpha^{+}(x) \wedge \mu^{\prime}(x), & \text { if } \bar{F}(\mu)(x) \geq \mu(x) \\ \alpha^{+}(x), & \text { if } \bar{F}(\mu)(x)<\mu(x)\end{cases} \\ \bar{F}(\mu)(0) & =\mu(0),\end{cases}
$$

and

$$
\begin{cases}\bar{B}(\mu)^{\prime}(x) & = \begin{cases}\alpha^{-}(x) \vee \mu^{\prime}(x), & \text { if } \bar{B}(\mu)(x) \geq \mu(x) \\ \alpha^{-}(x), & \text { if } \bar{B}(\mu)(x)<\mu(x)\end{cases} \\ \bar{B}(\mu)\left(s_{f}\right) & =\mu\left(s_{f}\right) .\end{cases}
$$

Proof. Let $J=\left\{x \in I: \operatorname{sign}\left(\mu^{\prime}-\alpha^{+}\right)\right.$is continuous at $\left.x\right\}$. Note that, since $\mu \in \mathscr{Q}, J$ contains almost all elements of $I$. Let $x \in J$, then

$$
\begin{gathered}
\lim _{h \rightarrow 0^{+}} \frac{\bar{F}(\mu)(x+h)-\bar{F}(\mu)(x)}{h}=\lim _{h \rightarrow 0^{+}} h^{-1}\left[\bigwedge_{y \leq x+h}\{\mu(y)+A(y, x+h)\}-\bar{F}(\mu)(x)\right]= \\
=\lim _{h \rightarrow 0^{+}} h^{-1}\left[\left(\bigwedge_{y \leq x}\{\mu(y)+A(y, x+h)\}-\bar{F}(\mu)(x)\right) \wedge\left(\bigwedge_{x<y \leq x+h}\{\mu(y)+A(y, x+h)\}-\bar{F}(\mu)(x)\right)\right]
\end{gathered}
$$

Since $A(y, x+h)=A(y, x)+A(x, x+h)$ by Proposition 5.4, the first parenthesis of (14) reduces to

$$
\bigwedge_{y \leq x}\{\mu(y)+A(y, x+h)\}-\bar{F}(\mu)(x)=\bar{F}(\mu)(x)+A(x, x+h)-\bar{F}(\mu)(x)=A(x, x+h) .
$$

Being $\operatorname{sign}\left(\mu^{\prime}-\alpha^{+}\right)$continuous at $x$, it is possible to choose $h>0$ sufficiently small such that $\operatorname{sign}\left(\mu^{\prime}-\alpha^{+}\right)$is constant on interval $[x, x+h]$. Set $J^{+}=\left\{x \in J: \mu^{\prime}(x)-\alpha^{+}(x)>0\right\}$ and $J^{-}=J \backslash J^{+}$. Then, the second parenthesis of (14) can be rewritten as

$$
\bigwedge_{x<y \leq x+h}\{\mu(y)+A(y, x+h)\}-\bar{F}(\mu)(x)= \begin{cases}\mu(x+h)-\bar{F}(\mu)(x), & \text { if } x \in J^{-} \\ \mu(x)+A(x, x+h)-\bar{F}(\mu)(x), & \text { if } x \in J^{+},\end{cases}
$$

since in the former case the minimum of $\mu(y)+A(y, x+h)$ over $[x, x+h]$ is attained at $x+h$, whilst in the latter is attained at $x$. 
Hence, we have that

$$
\begin{aligned}
\lim _{h \rightarrow 0^{+}} \frac{\bar{F}(\mu)(x+h)-\bar{F}(\mu)(x)}{h}= \begin{cases}\lim _{h \rightarrow 0^{+}} h^{-1}[A(x, x+h) \wedge(\mu(x+h)-\bar{F}(\mu)(x))], & \text { if } x \in J^{-} \\
\lim _{h \rightarrow 0^{+}} h^{-1}[A(x, x+h) \wedge(\mu(x)+A(x, x+h)-\bar{F}(\mu)(x))], & \text { if } x \in J^{+}\end{cases} \\
= \begin{cases}\alpha^{+}(x) \wedge \lim _{h \rightarrow 0^{+}} h^{-1}(\mu(x+h)-\bar{F}(\mu)(x)), & \text { if } x \in J^{-} \\
\alpha^{+}(x) \wedge \lim _{h \rightarrow 0^{+}} h^{-1}(\mu(x)+A(x, x+h)-\bar{F}(\mu)(x)), & \text { if } x \in J^{+}\end{cases} \\
= \begin{cases}\alpha^{+}(x) \wedge \mu^{\prime}(x), & \text { if } x \in J^{-} \text {and } \bar{F}(\mu)(x) \geq \mu(x) \\
\alpha^{+}(x) \wedge+\infty=\alpha^{+}(x), & \text { if } x \in J^{-} \text {and } \bar{F}(\mu)(x)<\mu(x) \\
\alpha^{+}(x)=\alpha^{+}(x) \wedge \mu^{\prime}(x), & \text { if } x \in J^{+} \text {and } \bar{F}(\mu)(x) \geq \mu(x) \\
\alpha^{+}(x) \wedge+\infty=\alpha^{+}(x), & \text { if } x \in J^{+} \text {and } \bar{F}(\mu)(x)<\mu(x)\end{cases} \\
= \begin{cases}\alpha^{+}(x) \wedge \mu^{\prime}(x), & \text { if } \bar{F}(\mu)(x) \geq \mu(x) \\
\alpha^{+}(x), & \text { if } \bar{F}(\mu)(x)<\mu(x) .\end{cases}
\end{aligned}
$$

Note that, by definition of $\bar{F}, \bar{F}(\mu)(x) \leq \mu(x)$ must hold. In conclusion, we proved that $\bar{F}(u) \in$ $W^{1, \infty}(I)$ and satisfies (12).

Applying the same reasoning it can be proved that $\bar{B}(u) \in W^{1, \infty}(I)$ and satisfies (13).

Proposition 5.10. Assume that $\mu^{+} \in \mathscr{Q}$ and let $w \in P$, then $w$ is feasible for Problem (5) (i.e., it satisfies constraints (5b) and (5c)), if and only if $\mu^{-} \leq w \leq \mu^{+}$and $\bar{M}(w)=w$.

Proof. $\Rightarrow)$ Assume that $w$ is feasible for Problem (5), then $w$ satisfies a. e. $w^{\prime} \leq \alpha^{+}$. Thus, $\phi=w$ is the solution of (6) for $\mu=w$, which implies that $\bar{F}(w)=w$. Analogously $\bar{B}(w)=w$, so that $\bar{M}(w)=w$. Moreover, since $w$ satisfies the bounds of Problem (5), it follows that $\mu^{-} \leq w \leq \mu^{+}$.

$\Leftarrow)$ Condition (5b) holds by hypothesis. Since $\bar{M}(w)=w$, then it must be $\bar{F}(w)=w$. In fact, if by contradiction $\bar{F}(w)<w, \bar{M}(w) \leq \bar{F}(w)<w$, which contradicts the assumption $\downarrow$. Then $\bar{F}(w)=w$, implies that, a. e., $\bar{F}(w)^{\prime}=w^{\prime}$ which by definition of $\bar{F}(w)^{\prime}$ in (6), implies that, a. e., $w^{\prime} \leq \alpha^{+}$. Analogously, it must be $\bar{B}(w)=w$ which implies that, a. e. $w^{\prime} \geq \alpha^{-}$and condition (5c) holds.

Proposition 5.11. Assume that $\mu^{+} \in \mathscr{Q}$. Then, Problem (5) is feasible if and only if $\bar{M}\left(\mu^{+}\right) \geq \mu^{-}$.

Proof. $\Rightarrow$ ) Let $w$ be a feasible solution of Problem (5). Then, by Proposition 5.10, $\bar{M}(w)=w \leq \mu^{+}$. Hence, being $\bar{M}$ order preserving, $\bar{M}\left(\mu^{+}\right) \geq \bar{M}(w) \geq \mu^{-}$.

$\Leftarrow) w=\bar{M}\left(\mu^{+}\right)$satisfies $\bar{M}(w)=w$ (by part ii. of Proposition 5.5) and $\mu^{+} \leq w \leq \mu^{-}$(by hypothesis). Hence, by Proposition 5.10, $w$ is a feasible solution of Problem (5).

Proposition 5.12. If $\mu^{+} \in \mathscr{Q}$ and Problem (5) is feasible, then $w^{*}=\bar{M}\left(\mu^{+}\right)$is its optimal solution.

Proof. By contradiction, assume that there exists a feasible $\tilde{w}$ such that $\Psi(\tilde{w})<\Psi\left(w^{*}\right)$. Since $\tilde{w}$ is feasible, Proposition 5.10 implies that $\bar{M}(\tilde{w})=\tilde{w}$. Moreover, since $\Psi$ is order reversing, $\tilde{w} \leq w^{*}$. This is not possible since $w^{*}=\bigvee\left\{w \in P: w \leq \bar{M}(w), w \leq \mu^{+}\right\} \geq \tilde{w}$, by Proposition 5.6 $\downarrow$. 


\section{References}

[1] F. Arscott and A. Filippov. Differential Equations with Discontinuous Righthand Sides: Control Systems. Mathematics and its Applications. Springer Netherlands, 2013.

[2] J. Bobrow, S. Dubowsky, and J. Gibson. Time-optimal control of robotic manipulators along specified paths. The International Journal of Robotics Research, 4(3):3-17, 1985.

[3] C. Chen, Y. He, C. Bu, J. Han, and X. Zhang. Quartic Bézier curve based trajectory generation for autonomous vehicles with curvature and velocity constraints. In Robotics and Automation (ICRA), 2014 IEEE International Conference on, pages 6108-6113, May 2014.

[4] L. Consolini, M. Locatelli, A. Minari, and A. Piazzi. A linear-time algorithm for minimum-time velocity planning of autonomous vehicles. In Proceedings of the 24th Mediterranean Conference on Control and Automation (MED), IEEE, 2016.

[5] L. Consolini, M. Locatelli, A. Minari, and A. Piazzi. An optimal complexity algorithm for minimum-time velocity planning. Systems \& Control Letters, 103:50-57, 2017.

[6] B. Davey and H. Priestley. Introduction to Lattices and Order. Cambridge University Press, 2002.

[7] M. Frego, E. Bertolazzi, F. Biral, D. Fontanelli, and L. Palopoli. Semi-analytical minimum time solutions for a vehicle following clothoid-based trajectory subject to velocity constraints. In 2016 European Control Conference (ECC), pages 2221-2227, June 2016.

[8] K. Kant and S. W. Zucker. Toward efficient trajectory planning: The path-velocity decomposition. The International Journal of Robotics Research, 5(3):72-89, 1986.

[9] T. Kunz and M. Stilman. Time-optimal trajectory generation for path following with bounded acceleration and velocity. Robotics: Science and Systems VIII, 2012.

[10] X. Li, Z. Sun, A. Kurt, and Q. Zhu. A sampling-based local trajectory planner for autonomous driving along a reference path. In Intelligent Vehicles Symposium Proceedings, 2014 IEEE, pages 376-381, June 2014.

[11] V. Muñoz, A. Ollero, M. Prado, and A. Simón. Mobile robot trajectory planning with dynamic and kinematic constraints. In Proc. of the 1994 IEEE Int. Conf. on Robotics and Automation, volume 4, pages 2802-2807, San Diego, CA, May 1994.

[12] Á. Nagy and I. Vajk. Lp-based velocity profile generation for robotic manipulators. International Journal of Control, pages 1-11, 2017.

[13] P. Shen, X. Zhang, and Y. Fang. Essential properties of numerical integration for time-optimal path-constrained trajectory planning. IEEE Robotics and Automation Letters, 2(2):888-895, April 2017.

[14] J. J. E. Slotine and H. S. Yang. Improving the efficiency of time-optimal path-following algorithms. IEEE Transactions on Robotics and Automation, 5(1):118-124, Feb 1989.

[15] R. Solea and U. Nunes. Trajectory planning with velocity planner for fully-automated passenger vehicles. In IEEE Intelligent Transportation Systems Conference, ITSC '06, pages $474-480$, September 2006. 
[16] E. Velenis and P. Tsiotras. Minimum-time travel for a vehicle with acceleration limits: Theoretical analysis and receding-horizon implementation. Journal of Optimization Theory and Applications, 138(2):275-296, 2008.

[17] D. Verscheure, B. Demeulenaere, J. Swevers, J. D. Schutter, and M. Diehl. Time-optimal path tracking for robots: A convex optimization approach. IEEE Transactions on Automatic Control, 54(10), Oct 2009.

[18] J. Villagra, V. Milanés, J. Pérez, and J. Godoy. Smooth path and speed planning for an automated public transport vehicle. Robotics and Autonomous Systems, 60:252-265, 2012. 